\title{
Estimation of a super-resolved PSF for the data reduction of undersampled stellar observations
}

\section{Deriving an accurate model for fitting photometry with Corot space telescope}

\author{
L. Pinheiro da Silva ${ }^{1,2}$, M. Auvergne ${ }^{3}$, D. Toublanc ${ }^{2}$, J. Rowe ${ }^{4}$, R. Kuschnig ${ }^{4}$, and J. Matthews ${ }^{4}$ \\ 1 Centre National d'Études Spatiales (CNES), 18 Av. Édouard Belin, 31401 Toulouse, France \\ e-mail: leonardo.pinheirodasilva@cesr.fr \\ 2 Centre d'Étude Spatiale des Rayonnements (CESR/CNRS), 7 Av. du Colonel Roche, 31077 Toulouse, France \\ 3 Observatoire de Paris-Meudon (LESIA/CNRS), 5 place Jules Janssen, 92195 Meudon, France \\ ${ }^{4}$ Department of Physics and Astronomy, University of British Columbia, 6224 Agricultural Road, Vancouver, Canada
}

Received 30 November 2005 / Accepted 9 February 2006

\begin{abstract}
Context. Fitting photometry algorithms can be very effective provided that an accurate model of the instrumental point spread function (PSF) is available. When high-precision time-resolved photometry is required, however, the use of point-source star images as empirical PSF models can be unsatisfactory, due to the limits in their spatial resolution. Theoretically-derived models, on the other hand, are limited by the unavoidable assumption of simplifying hypothesis, while the use of analytical approximations is restricted to regularly-shaped PSFs.

Aims. This work investigates an innovative technique for space-based fitting photometry, based on the reconstruction of an empirical but properly-resolved PSF. The aim is the exploitation of arbitrary star images, including those produced under intentional defocus. The cases of both MOST and COROT, the first space telescopes dedicated to time-resolved stellar photometry, are considered in the evaluation of the effectiveness and performances of the proposed methodology.

Methods. PSF reconstruction is based on a set of star images, periodically acquired and presenting relative subpixel displacements due to motion of the acquisition system, in this case the jitter of the satellite attitude. Higher resolution is achieved through the solution of the inverse problem. The approach can be regarded as a special application of super-resolution techniques, though a specialised procedure is proposed to better meet the PSF determination problem specificities. The application of such a model to fitting photometry is illustrated by numerical simulations for COROT and on a complete set of observations from MOST.

Results. We verify that, in both scenarios, significantly better resolved PSFs can be estimated, leading to corresponding improvements in photometric results. For COROT, indeed, subpixel reconstruction enabled the successful use of fitting algorithms despite its rather complex PSF profile, which could hardly be modeled otherwise. For MOST, whose direct-imaging PSF is closer to the ordinary, comparison to other models or photometry techniques were carried out and confirmed the potential of PSF reconstruction in real observational conditions.
\end{abstract}

Key words. techniques: photometric - methods: data analysis - space vehicles: instruments - stars: oscillations

\section{Introduction}

The reduction of astronomical data often requires a model representing the instrumental point spread function (PSF). That is typically the case, for instance, when fitting algorithms are selected for the precise determination of stellar coordinates and intensities, i.e., in astrometric or photometric applications. Given the optics design, such a PSF model can be in principle produced by numerical simulations, but it is generally difficult to match the many physical parameters of the instrument on which the PSF is significantly dependent. Moreover, real-world PSFs use to be quite complex and unaccounted-for phenomena may lead even sophisticated computational derivations to be less realistic than desirable. Alternatively, point-source star images can be seen as rather fair PSF representations, in a sense, but their spatial resolution is limited by the physical characteristics of the imaging system (e.g., the pixel size of CCD detectors). That resolution can be insufficient when very precise measurements are desired, on multiple observations over time. Indeed, under inadequate resolution, the intensity profiles of acquired images turn out to be strongly dependent on the actual intra-pixel location of the star on the focal plane. In this case, the PSF must be modeled with subpixel accuracy if scientific parameters are to be accurately estimated with no biasing between different observations.

In the present work, the purpose has been the estimation of a properly resolved PSF model enabling the use of fitting algorithms on data from space photometry missions. Particularly, the presented methodology has been studied in the framework of the COROT mission ${ }^{1}$, to be launched in 2006. In complement, validation and tests were made possible by real observations from

${ }^{1}$ COROT (COnvection, ROtation and planetary Transits) is a space mission led by the French Space Agency with the collaboration of many european countries and Brazil. Its main scientific goals are the study of stellar variations and the detection of extrasolar planets. 

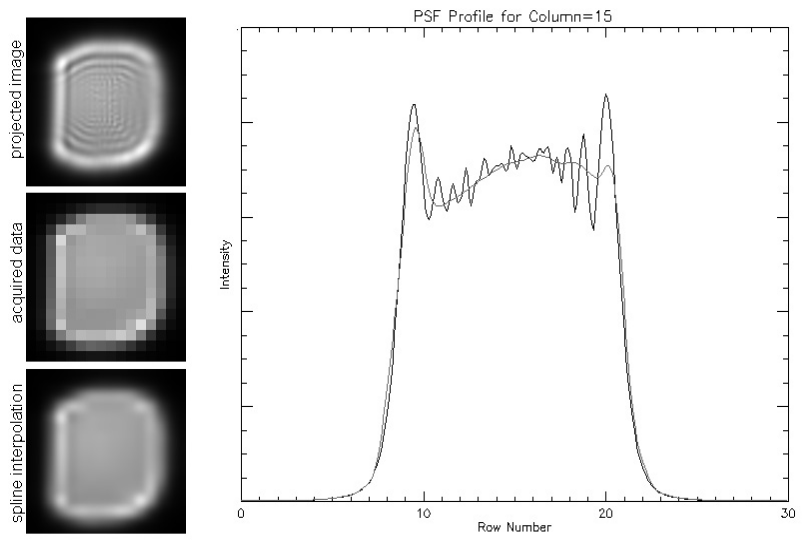

Fig. 1. The defocused Corot PSF as projected on the CCD surface (top), the acquired data after downsampling by the pixel lattice (middle), and the cubic interpolation of these data for a smooth PSF representation (bottom). Horizontal slices for the top and bottom images are shown on the right panel, for better comparison.

MOST space telescope ${ }^{2}$, in orbit since 2003. Both are scientific micro-satellites designed to perform high-precision stellar photometry from space, and this paper was made possible by a general cooperation agreement between MOST and COROT teams.

PSF fitting has been already extensively studied and routinely applied to stellar photometry mainly in crowded fields, aperture photometry being usually preferred otherwise. In the first case, PSF models are generally obtained through the adjustment of analytical (e.g. Gaussian or Moffat) profiles to the images, sometimes along with a numeric description of the residuals. In time-resolved space photometry, however, the employment of fitting algorithms can be justified even for isolated stars, but alternative PSF determination techniques may be required in this case. Firstly, those algorithms can enable a better exploitation of many classes of noisy or degraded images, present lower sensitivity to periodic disturbances such as stray light, and provide some robustness specially against the occurrence of particle impacts. That is particularly interesting for missions in low Earth orbit like COROT and MOST, which are to spend roughly 5 to $10 \%$ of their lifetime crossing the South Atlantic Anomaly. Secondly, it has been shown that even in the case of non-degraded images of isolated stars, best performances can always be achieved by combined aperture/fitting photometry algorithms (Kjeldsen \& Frandsen 1992), making a PSF model desirable in any case.

Since photometry-dedicated instruments need not to be infocus, both COROT and MOST telescopes were designed to spread the incoming star light over a larger CCD area, for better adaptation to technical aspects such as pixel saturation and pixel response non-uniformity. We verify, as shown in Fig. 1, that quite complex PSFs may result in this case. The adjustment of classic analytical functions to those profiles is clearly unsuitable, an empirical model being required instead. When such a PSF is acquired by a CCD, on the other hand, the resulting model is undersampled, in the sense that the sampling resolution was insufficient for the proper registration of its finest spatial features. That includes the generation of spurious asymmetries dependent on the exact subpixel position of the image on the pixel lattice. If one considers a realistic set of exposures, aimed at time-resolved

${ }^{2}$ MOST (Microvariability and Oscillations of STars) is a Canadian Space Agency mission, operated jointly by Dynacon, Inc., and the Universities of Toronto and British Columbia, with the assistance of the University of Vienna. photometry, it is clear that none of the acquired profiles, nor a linear combination of many, would therefore constitute a proper model for PSF fitting. For a generic model enabling precise and robust fitting photometry, the derivation of an empirical but better resolved PSF from a set of low-resolution star images is investigated in this work. In general, techniques aiming at the restitution of high resolution data from a set of decimated images are gathered under the domain of super-resolution theory. Under certain conditions, such a restitution is possible, at least in principle, through the exploitation of subpixel motion occurring between the camera and the target during distinct image acquisitions, as in the case of an imaging satellite presenting some level of attitude jitter, for instance.

Although generic and well-known super-resolution algorithms may work fine in the reconstruction of ordinary scenes, they are not optimised to the special case of star images. That is because, in most cases, these algorithms assume a degree of smoothness of the solution which does not apply to a PSF model.In other cases, they are based on hypotheses about the sampling process or the relative image displacements that are not quite valid in the scenarios of our interest. For the reconstruction of a sharp-edged PSF from space-based observations, a better suited procedure is therefore proposed and evaluated. Super-resolution fundamentals are described in Sect. 2, and the proposed methodology is described in Sect. 3. PSF reconstruction and photometry tests for COROT are presented in Sect. 4. Application to fitting photometry with MOST is discussed in Sect. 5, and conclusions are presented in Sect. 6.

\section{Background}

The classic approach for the reconstruction of a super-resolved image through the inversion of an observation model is briefly reviewed in this section. Mathematical derivations are presented in the Appendix, along with further details. For introductory material, the reader is referred to many well-written texts available on subject (see for example Park et al. 2003; Elad \& Feuer 1997).

Let $X$ represent an image (or scene) of arbitrary resolution, which is supposed to be registered by an acquisition system. In the presence of relative motion of the imaging device (with respect to the scene) between distinct acquisitions, and considering a limited spatial resolution of its detector (e.g, a CCD), each acquired image $Y_{k}$ may be described as

$Y_{k}=D W_{k} X+\eta_{k} \quad(k=1, . ., N)$.

Here, $W_{K}$ represents the geometric transformation presented by each image due to relative motion, supposed to be known, and $D$ represents the spatial downsampling performed by the detector. The noise intrinsic to the registration process is represented by $\eta$. Images $X$ and $Y_{k}$ are arranged as vectors in lexicographic form.

Super-resolution theory deals with the solution of the inverse problem. Given a set of low-resolution images $Y_{k}$, and supposing that $D$ and $W_{k}$ are known, we are interested in the estimation of a higher-resolution image $X$. Let $\hat{X}$ be for instance the estimate in a least-square basis, which minimizes the cost function $\phi$ defined as

$\phi=\sum_{k=1}^{N}\left[Y_{k}-D W_{k} X\right]^{T}\left[Y_{k}-D W_{k} X\right]$ 
The theoretical solution can be derived from the above expression by equaling its first derivative to zero, leading to the matrix equation

$\sum_{k=1}^{N}\left[W_{k}^{T} D^{T} D W_{k}\right] \cdot \hat{X}=\sum_{k=1}^{N}\left[W_{k}^{T} D^{T} Y_{k}\right]$

Unfortunately, the direct solution of this equation is rarely possible. The super-resolution problem is known to be ill-posed in general, so that a unique solution not necessarily exists, and the matrix on the left side is most often singular. Moreover, matrix dimensions grow up very quickly with the target resolution, leaving the inverse operation computationally infeasible in any case.

These issues are usually addressed in two steps. First, to stabilize the solution, a regularization term is introduced in the cost function,

$\phi=\sum_{k=1}^{N}\left\|Y_{k}-D W_{k} X\right\|^{2}+\lambda\|C X\|^{2}$,

where $C$ is most often an operator penalizing high-frequencies in the reconstructed images, thus favoring smoother solutions, and $\lambda$ is an adjustable regularization parameter. The previous equation then becomes

$\sum_{k=1}^{N}\left[W_{k}^{T} D^{T} D W_{k}+\lambda C^{T} C\right] \cdot \hat{X}=\sum_{k=1}^{N}\left[W_{k}^{T} D^{T} Y_{k}\right]$.

Second, an iterative algorithm is employed in the solution of this equation, to enable a practical implementation despite the matrix dimensions involved. A common choice is the gradient-descent, defined in this case as

$\hat{X}_{j+1}=\hat{X}_{j}+\mu \sum_{k=1}^{N}\left[W_{k}^{T} D^{T} Y_{k}\right]-\left[W_{k}^{T} D^{T} D W_{k}+\lambda C^{T} C\right] \hat{X}_{j}$,

where $\mu$ is a user-defined convergence step.

\section{Methodology}

The algorithm described in the previous section represents the most classic regularized solution to the problem of restoring high-frequency features from an image set. In the last years, many variants have evolved in search for better computational performance, or aiming to overcome some of its intrinsic limitations. For PSF reconstruction, particularly, computational performance is not critical, since individual star images are typically small and a simple algorithm is usually enough for the achievement of convergence in a reasonable amount of time. On the other hand, an alternative regularization method is indeed necessary if sharp PSF edges are to be preserved and properly restituted.

For an estimation aiming at time-resolved fitting photometry, an effective but not overcomplicated choice is to use a regularization term based on the comparison between the solution candidates $\hat{X}_{j}$ and a decimated but sharp-edged PSF estimate denominated $\hat{X}_{0}$. That corresponds to the minimization of

$\phi=\sum_{k=1}^{N}\left\|Y_{k}-D W_{k} X\right\|^{2}+\lambda\left\|X-X_{0}\right\|^{2}$.

The later parameter is computed a-priori as the average of all input images, after recentering for motion compensation and cubic interpolation to match the target resolution. That parameter is called $\hat{X}_{0}$ for it seems interesting to use it as start-point in the recursive process, for faster results, although that is not a requirement for convergence. It can be shown (cf. Appendix) that the corresponding recursive rule is

$\hat{X}_{j+1}=\hat{X}_{j}+\mu \sum_{k=1}^{N}\left[W_{k}^{T} D^{T} Y_{k}\right]-\left[W_{k}^{T} D^{T} D W_{k}\right] \hat{X}_{j}-\lambda\left[\hat{X}_{j}-\hat{X}_{0}\right]$,

where $\mu$ controls the convergence speed and $\lambda$ enables a compromise between the rejection of artifacts and the fidelity of the solution to the input data.

Fortunately, given the observation of a point-source star, we verify that each transformation matrix $W_{k}$ turns out to represent a pure translational motion of the solution image $X$. That is true even in the case of rotation of the imaging instrument along the line of sight, and whatever the shape of the PSF. That amount of translation of the target star for each image $Y_{k}$, given in pixels in both directions with respect to an arbitrary reference, must be known with subpixel accuracy for PSF reconstruction. It may be algebraically derived from satellite attitude data or directly deduced from the acquired images themselves. The matrix $D$, in the case of a CCD detector, stands for the flux integration performed over each pixel surface, and can be seen as a nonideal downsampling operator which performs the summation of adjacent values in $X$ to yield a lower resolution image $Y_{k}$.

Although those matrices are necessary for theoretical derivations, there is actually no need for their construction in practice. Indeed, for a computational implementation of the algorithm, all transformations can be conveniently approximated by pixelwise operations. The computation of pure translations $(W)$ and downsampling operations $(D)$ by image manipulation is straightforward. It can also be verified that the multiplication by the transposed matrix $W_{k}^{\mathrm{T}}$ can be substituted by a translation in the opposed direction with respect to $W_{k}$, and that the role played by $D^{\mathrm{T}}$ can be implemented by an image rescale with no interpolation.

Finally, it must be noted that proper reconstruction requires some degree of pre-processing of the individual images, which may have significant impact on the quality of the resulting PSF. The most critical treatments are for the correction of potentially time-varying contributions, such as the sky background and the electronic bias, which might lead to unequal image weighting if not accounted for. The normalization of the corrected images may also be desirable.

\section{Application to COROT simulated data}

\subsection{PSF reconstruction}

We illustrate the application of the proposed procedure to COROT (for details about the instrument and mission profile, see Boisnard \& Auvergne 2004). The exploitation of stellar images from its seismology field is analysed. As seen in Fig. 1, the corresponding PSF is significantly defocused and can spread over 400 pixels or more.

Input data to the algorithm were provided by a set of 500 synthetic images, comparable in number and quality to what is to be acquired by COROT in less than 3 orbits. Images were generated basically through successive operations of translation and downsampling of a theoretical high-resolution PSF, issued from modeling of the instrument optics. The resulting profiles were scaled to accommodate a total of $1.08 \times 10^{7}$ counts each, corresponding to the collected flux for one of the faintest COROT primary targets $\left(m_{\mathrm{v}}=9 ; T_{\text {eff }}=6000 \mathrm{~K}\right)$ in $32 \mathrm{~s}$ of integration time, given 
the pupil surface and transmissions. This scenario was selected for representing the noisiest and thus most pessimistic case for PSF estimation.

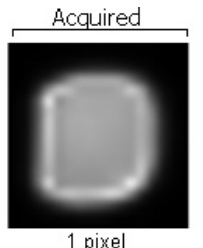

1 pixel

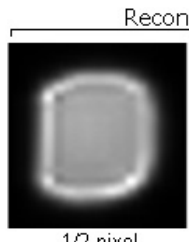

$1 / 2$ pixel

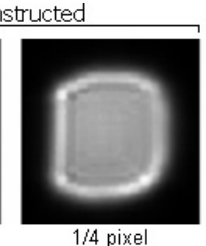

$1 / 4$ pixel

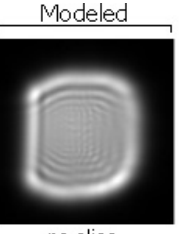

no alias
Fig. 2. Acquired, reconstructed and modeled PSFs for Corot.

The exact amount of translation was unique for each image and such as to correspond to the expected motion of the target star, with respect to the pixel grid, due to jitter of the satellite attitude, which is in the range of \pm 0.2 pixel in each axis. Downsampling was performed through integration of the PSF over each pixel area, and Poisson and readout noises (the later of 10 counts per pixel per readout in average) were at last introduced.

The estimation of the relative displacements presented by each image, which is also required as an input for the restoration algorithm, was evaluated simply as the difference between each image barycentre and an arbitrary reference. That was preferred although more precise estimates will be available in COROT telemetry, for simplicity and as a mean of robustness verification.

In Fig. 2, two reconstructed PSFs are shown, for doubled and four-folded resolutions. Cubic interpolation of a typical (simulated) image acquisition is also shown for comparison, along with the original PSF model employed on image simulations.

We verify the proper retrieval of the major spatial structures and the restoration of originally decimated symmetries. That is better illustrated by vertical and horizontal PSF slices shown in Fig. 3, specially if compared to Fig. 1. The effectiveness of these reconstructed models when employed on fitting photometry is analysed in the next section.
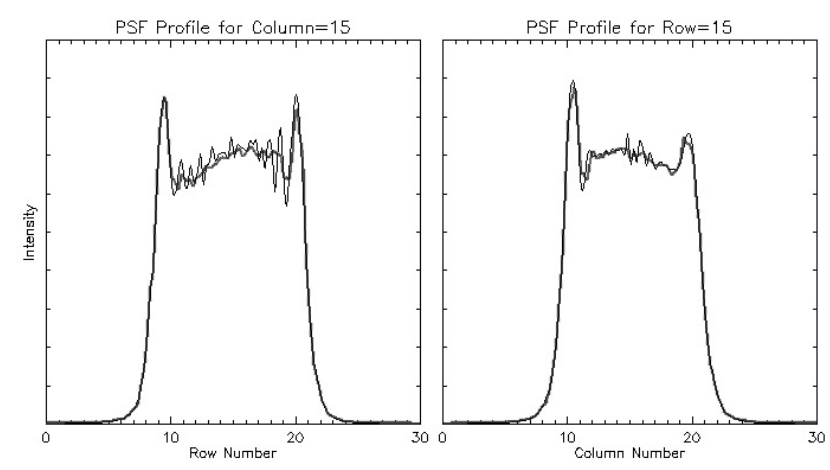

Fig. 3. Slices of reconstructed (1/4 pixel) and modeled PSFs for Corot.

\subsection{Fitting photometry}

An implementation of Levenberg-Marquardt algorithm was employed to fit any of the available PSF models to a given set of stellar images. Four variables are let free to converge in this fitting process, as follows. First, the photometric measurement itself is represented by a pure multiplicative factor applied to the PSF. Second, a uniform background level is accounted for by means of a constant value which is added to the scaled model. Finally, two variables, $x$ and $y$, representing the exact PSF position in the image, enable it to be freely translated for error minimization.

For evaluation purposes, fitting was performed on synthetic sets of images generated as described in the later section. Nevertheless, different displacements and uncorrelated noises were introduced, with respect to those simulated for PSF reconstruction, to avoid biased results. Two stellar magnitudes were considered, $m_{\mathrm{v}}=6$ and 9 , which represent roughly the extremes of interest for the COROT central program. Approximately 2000 images were processed in each case, corresponding to COROT observations during 10 orbital periods. No flux variation was introduced, so that any variation of the photometric signal could be promptly identified as noise or artifact.

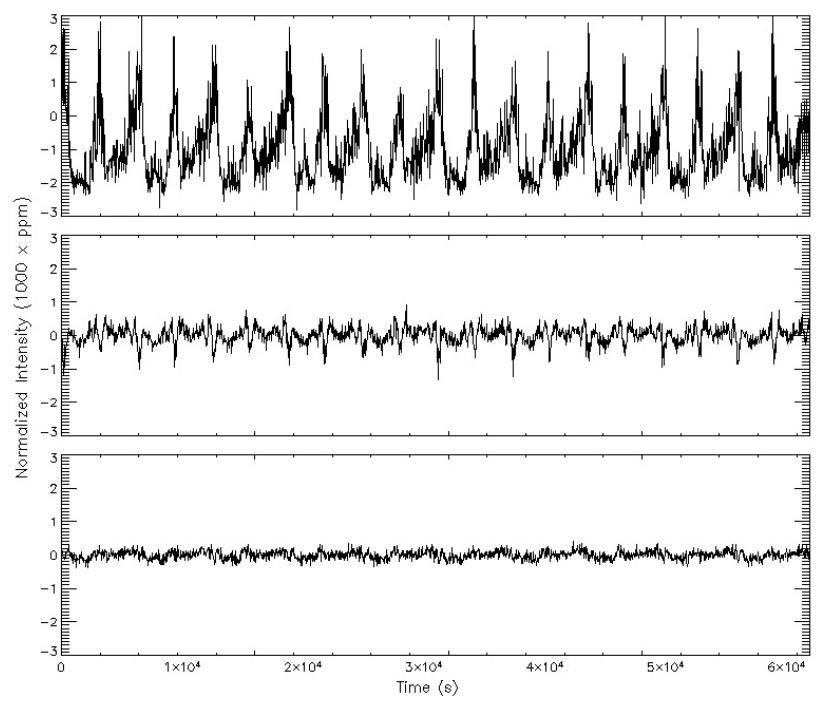

Fig. 4. Fitting photometry on simulated COROT images, for three different PSF models (from top to bottom): a 1-pixel resolution PSF directly extracted from stellar images, and two reconstructed PSFs at the resolution of half and a quarter of a pixel.

We present in Fig. 4 the resulting photometric signal when three different PSFs are used as input model for fitting. Data for the brightest magnitude are shown, since its photon noise is minimal and artifacts can be better appreciated. Initially, an undersampled PSF is directly derived from the available images, through compensation of the relative motion (recentering) and averaging. In this case, as discussed, we verify that insufficient resolution leads to uneven measurements for different subpixel positions of the star. The signal is hence significantly disturbed in response to variations of the satellite attitude, specially at the orbital period and its harmonics. That effect is progressively reduced, though, when the previously reconstructed PSFs are employed, at doubled and four-folded resolutions respectively. If a residue is still visible for a resolution of $1 / 2$ pixel, we verify that periodic artifacts are just comparable to the white noise level when the best resolved PSF reconstruction at $1 / 4$ pixel is used.

With respect to the white noise itself, a comparison for each PSF model and stellar magnitude is presented in Table 1. We verify that white noise level also falls abruptly with the use of reconstructed PSFs. For $m_{\mathrm{v}}=9$, it turns out that noise levels are quickly dominated by photon noise, even for a PSF resolved at just half of a pixel. For $m_{\mathrm{v}}=6$, a noise level as low as $16 \%$ 
Table 1. Fitting photometry error for four PSF models.

\begin{tabular}{ccc}
\hline \hline PSF model & $m_{\mathrm{v}}=6$ & $m_{\mathrm{v}}=9$ \\
\hline Recenter-and-average & $4.99 \times 10^{-4}$ & $5.50 \times 10^{-4}$ \\
Reconstruction at 1/2 pixel & $1.43 \times 10^{-4}$ & $3.41 \times 10^{-4}$ \\
Reconstruction at 1/4 pixel & $9.14 \times 10^{-5}$ & $3.24 \times 10^{-4}$ \\
Ideal (no PSF uncertainty) & $7.83 \times 10^{-5}$ & $3.19 \times 10^{-4}$ \\
\hline
\end{tabular}

above the photon noise could be achieved with the use of the best resolved PSF reconstruction.

\section{Application to MOST observations}

\subsection{PSF reconstruction}

In complement to numerical simulations presented in the previous section, reconstruction tests have been carried out on a complete set of MOST observations, acquired from its direct imaging field (for details, see Walker et al. 2003). Because of its less aggressive defocus, these MOST images were much sharper than those expected for COROT, leading to even greater decimation due to undersampling. The CCD pixel size is very similar for both instruments, and PSF reconstruction was again chosen to be performed at the resolution of $1 / 4$ of a pixel, enabling direct comparisons with the simulated results already discussed.

Nearly 32 days of observations of the secondary MOST target HD $61199\left(m_{\mathrm{v}}=8\right)$ were employed, corresponding to roughly 100000 images. From this data set, just 1000 images were selected for PSF determination, and the remaining data was left for an extensive validation of the resulting model. The selection criteria were the level of stray light and the absence of particle impacts, so that the less noisy images were exploited in the reconstruction phase.

The detected star flux was of approximately $6.0 \times$ $10^{4}$ counts per image in average, a greater Poisson noise implying a more severe condition for PSF reconstruction than that presented in the previous section. That is partly due to a much shorter exposure time $(0.9 \mathrm{~s})$. Before application of the reconstruction algorithm, images were corrected for sky background and the electronic bias, and then normalised. Relative motion was directly derived from variations of the barycentre of the star.

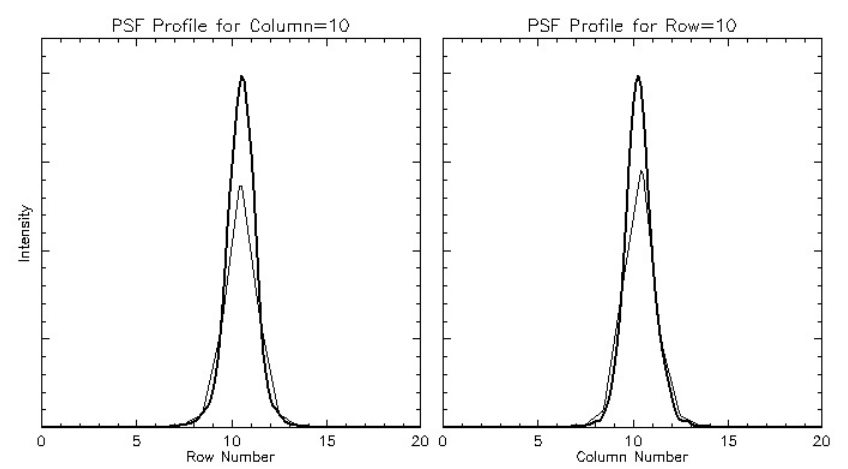

Fig. 5. Slices of reconstructed PSF for Most, as compared to 1-pixel data.

The resulting PSF estimate is shown in Fig. 5, in comparison to a typical image acquisition resolved at the CCD pixel size. We verify the evaluation of a narrower and significantly sharper profile, leading to a presumably less aliased PSF. For the purposes of this work, we are mainly interested in its ability to perform as an accurate model for fitting photometry, which is investigated in the next section.

\subsection{Fitting photometry}

Photometric measurements were performed on the whole data set available for HD 61199. That star turns out to be a multiperiodic variable (Matthews et al. 2004) and thus constitutes a very interesting target for time-resolved photometry evaluation.

In addition to the fitting of the empirical PSF previously derived, three concurrent methodologies were selected for comparison: two classic algorithms based on a threshold or aperture, and the fitting of a Moffat analytical profile. The empirical profile was fit with use of the same algorithm and methodology described in Sect. 4.2.

To avoid any biasing as a consequence of data reduction, algorithms were applied to raw stellar images and very basic post-processing was equally applied to all resulting signals. That processing consisted of outliers rejection (mainly due to particle impacts or excessive satellite depointing), decorrelation with respect to the sky background, and detrending to account for low-frequency instrumental phenomena. That is certainly suboptimal for the generation of scientific data through any of the considered algorithms, but enables their intrinsic characteristics to be appreciated before being masked by further processing steps.

Results for each photometric procedure are shown in Fig. 6, and the effects of the incomplete data reduction are mostly visible as periodic artifacts at the orbital period and harmonics (the corresponding frequencies are indicated in the $x$-axis, in the figure). As antecipated in the Introduction section, these results illustrate the interest for fitting algorithms in comparison to other data reduction techniques, for space-based time-resolved photometry. We verify that threshold photometry was greatly

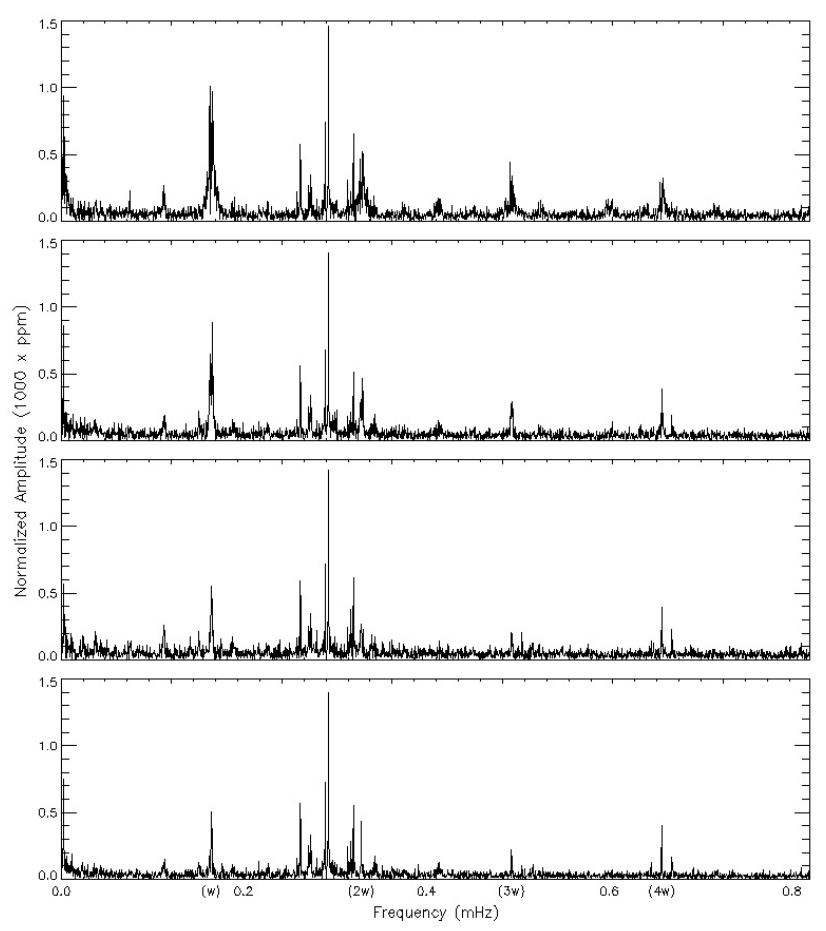

Fig. 6. Amplitude spectrum of MOST's secondary target HD 61199, as evaluated by four photometry algorithms. From top to bottom: threshold photometry, aperture photometry, fitting of a Moffat profile, and fitting of the empirical PSF reconstructed at $1 / 4$ of a pixel. 
Table 2. White noise level for different photometry techniques.

\begin{tabular}{ccc}
\hline \hline Technique & $\sigma_{\text {photometry }}$ & $\sigma_{\text {photo }} / \sigma_{\text {Poisson }}$ \\
\hline Threshold photometry & $7.66 \times 10^{-3}$ & 1.876 \\
Aperture photometry & $7.03 \times 10^{-3}$ & 1.721 \\
Fitting of Moffat profile & $7.36 \times 10^{-3}$ & 1.802 \\
Fitting of reconstructed PSF & $4.89 \times 10^{-3}$ & 1.197 \\
\hline
\end{tabular}

disturbed by periodic variations of the stray light level, leading to many degraded frequency components. Synthetic-aperture, despite its effectiveness in the reduction of isolated observations, is rather sensitive to motion of the target star over the CCD surface, and was affected in this case by jitter of the satellite attitude.

With respect to the alternative PSF fitting techniques, performances can be compared in two different aspects. First, we verify that both models led to very similar results regarding the restitution of frequency components in evidence, either scientific (particularly the one representing the main stellar pulsation) or technological. Those which are modulated at the orbital period are constrained to very narrow frequency bands, in contrast to threshold or aperture signals, and would be easily corrected for. In terms of the white noise level, however, better performances were achieved when the reconstructed PSF model was employed. In this case, noise level turned out to be just $19 \%$ above the theoretical photon noise (even without taking the contribution of stray light into account). The fitting of a Moffat profile, on the other hand, led to no significant improvement in white noise level in comparison to other photometric procedures (roughly $80 \%$ above photon noise). All those quantitative results are summarised in Table 2, as estimated from relative photometric signals.

Considering that a Moffat profile already mimics MOST's PSF somewhat closely, the fact that it could be matched and even exceeded by an empirical model confirms the potential of the reconstruction method applied to high-precision photometry by fitting. If a sub-pixel reconstruction is not actually indispensable for regular PSFs, and indeed both models led to satisfactory results in this case (as shown in Fig. 6), such a reconstruction can be required whenever an analytical profile is not applicable, as illustrated for COROT.

\section{Conclusion}

This work has analysed the interest in reconstructing a highresolution empirical PSF for the reduction of astronomical data. We verified that the application of super-resolution techniques to stellar images can result in accurate PSF models, which can be then employed on fitting algorithms aiming at the extraction of astronomical signals. This data reduction methodology has been illustrated by time-resolved photometry tests in two complementary scenarios. First, in the case of the COROT mission, a rather complex PSF is expected and an empirical model was thus required. Through numerical simulations, we verified that a reconstructed model at $1 / 4$ of a pixel was indeed possible, and allowed a significant improvement in the measurement of photometric signals. Second, in the case of the MOST mission, real observational data from its direct imaging field were employed, from which a more classic PSF profile is obtained. That enabled the application of a reconstructed high-resolution PSF to be compared with that of a Moffat profile, a widely used analytical model. We verified that the results obtained with the later could be matched and even exceeded by the former. Based on both results, we conclude that the procedure for PSF determination proposed in this paper can be considered whenever fitting algorithms are to be employed on the reduction of astronomical images, if extreme accuracy is required. Moreover, it can extend the applicability of those algorithms, such as in the case of COROT, thanks to the greater generality owed to its empirical character.

Acknowledgements. This work was originally made possible by Fuad Kassab $\mathrm{Jr}$ (EP/USP), Eduardo Janot Pacheco (IAG/USP), Annie Baglin (LESIA) and Alain Gaboriaud (CNES). We are also grateful to Réza Samadi (LESIA), Frédéric Baudin (IAS) and Laurent Boisnard (CNES) for the valuable discussions and support. LPS is funded by the Brazilian Ministry of Science and Technology (CNPq grant \#200334/2003-4).

\section{Appendix A: Regularized image reconstruction}

Consider the reconstruction of a single high-resolution image, represented by $X$, given a set of $N$ low-resolution images acquired with different subpixel displacements of the camera. Each acquired image $Y_{k}$ may be described as

$Y_{k}=D W_{k} X+\eta_{k} \quad(k=1, . ., N)$,

where:

- $X$ is a [N1.N2] vector representing a [N1 $x N 2]$ image;

- $Y_{k}$ is a [M1.M2] vector representing a [M1 $x$ M2] image;

- $W_{k}$ is a geometric warp matrix of size [N1.N2 $\left.x N 1 . N 2\right]$;

- $D$ is the downsampling matrix of size [M1.M2 $\times N 1 . N 2]$;

- $\eta_{k}$ is a $M 1 . M 2$-element vector representing the noise for each image acquisition.

Images $X$ and $Y_{k}$ are arranged as vectors through the stacking of successive columns. Matrix $W_{k}$ represents the transformation of the high-resolution image $X$ into its shifted/rotated version, as seen by the camera due to its relative motion. Matrix $D$ downsamples the resulting image $W_{k} X$, leading to the acquired image $Y_{k}$.

In a first approach for the solution of the inverse problem, let $\hat{X}$ be the estimate which minimizes the cost function defined as

$\phi_{1}=\sum_{k=1}^{N}\left\|Y_{k}-D W_{k} X\right\|^{2}=\sum_{k=1}^{N}\left[Y_{k}-D W_{k} X\right]^{T}\left[Y_{k}-D W_{k} X\right]$.

Making the first derivative (with respect to $X$ ) equal to zero, and isolating $X$, leads to:

$\sum_{k=1}^{N}\left[2 W_{k}^{T} D^{T} D W_{k} \hat{X}-2 W_{k}^{T} D^{T} Y_{k}\right]=0$

$\sum_{k=1}^{N}\left[W_{k}^{T} D^{T} D W_{k}\right] \cdot \hat{X}=\sum_{k=1}^{N}\left[W_{k}^{T} D^{T} Y_{k}\right]$

In general, the image reconstruction problem is underestimated and there are infinite solutions satisfying the above equation. The use of regularized cost function is therefore necessary instead, such as the Tikhonov function defined as

$\phi_{2}=\sum_{k=1}^{N}\left\|Y_{k}-D W_{k} X\right\|^{2}+\lambda\|C X\|^{2}$

where $C$ represents any operator behaving as a high-pass filter, such as the discrete Laplacian matrix (Elad \& Feuer 1997). The parameter $\lambda$ is user-defined and enables more or less aggressive regularization to be imposed (smoothness of the solution versus fidelity to the input data). 
In an analogous way, the expression for the estimate $\hat{X}$ minimizing $\phi_{2}$ becomes:

$$
\begin{aligned}
& \sum_{k=1}^{N}\left[2 W_{k}^{T} D^{T} D W_{k} \hat{X}-2 W_{k}^{T} D^{T} Y_{k}+2 \lambda C^{T} C \hat{X}\right]=0 \\
& \sum_{k=1}^{N}\left[W_{k}^{T} D^{T} D W_{k}+\lambda C^{T} C\right] \cdot \hat{X}=\sum_{k=1}^{N}\left[W_{k}^{T} D^{T} Y_{k}\right] .
\end{aligned}
$$

Finally, considering the large matrix dimensions involved, the solution of the above equation is better achieved through the use of an iterative algorithm. The best known is probably the Landweber iteration (Calvetti et al. 1998) expressed in this case by

$\hat{X}_{j+1}=\hat{X}_{j}+\mu \sum_{k=1}^{N}\left[W_{k}^{T} D^{T} Y_{k}\right]-\left[W_{k}^{T} D^{T} D W_{k}+\lambda C^{T} C\right] \hat{X}_{j}$,

where $\mu$ is a convergence step.

An alternative cost function whose regularization is based on a reference image, thus allowing for the existence of strong gradients in the solution, can be expressed by

$\phi_{3}=\sum_{k=1}^{N}\left\|Y_{k}-D W_{k} X\right\|^{2}+\lambda\left\|X-X_{0}\right\|^{2}$

Once more, the expression for $\hat{X}$ can be derived from the first derivative of $\phi_{3}$, as follows:

$$
\begin{aligned}
& \sum_{k=1}^{N}\left[2 W_{k}^{T} D^{T} D W_{k} \hat{X}-2 W_{k}^{T} D^{T} Y_{k}+2 \lambda\left(\hat{X}-X_{0}\right)\right]=0 \\
& \sum_{k=1}^{N}\left[W_{k}^{T} D^{T} D W_{k}+\lambda I\right] \cdot \hat{X}=\sum_{k=1}^{N}\left[W_{k}^{T} D^{T} Y_{k}+\lambda X_{0}\right],
\end{aligned}
$$

where $I$ is the identity matrix.
The corresponding Landweber iteration is given by

$$
\hat{X}_{j+1}=\hat{X}_{j}+\mu \sum_{k=1}^{N}\left[W_{k}^{T} D^{T} Y_{k}+\lambda X_{0}\right]-\left[W_{k}^{T} D^{T} D W_{k}+\lambda I\right] \hat{X}_{j},
$$

or, re-arranging:

$\hat{X}_{j+1}=\hat{X}_{j}+\mu \sum_{k=1}^{N}\left[W_{k}^{T} D^{T} Y_{k}\right]-\left[W_{k}^{T} D^{T} D W_{k}\right] \hat{X}_{j}-\lambda\left[\hat{X}_{j}-\hat{X}_{0}\right]$.

\section{References}

Anderson, J., \& King, I. R. 2000, PASP, 112, 1360

Boisnard, L., \& Auvergne, M. 2004, Corot Mission Engineeging: Cost-Effective Solutions for Stellar Photometry in Low Earth Orbit. 55th Congress of the International Astronautical Federation (IAF), IAC-04-IAF-Q.1.01

Borman, S., \& Stevenson, R. 1998, Spatial Resolution Enhancement of Low-Resolution Image Sequences - A Comprehensive Review. Technical Report, University of Notre Dame, USA

Calvetti, D., Reichel, L., \& Zhang, Q. 1998, Applied Comput. Control, Signals and Circuits, 1,317

Cheeseman, P., Kanefsky, B., Kraft, R., Stutz, J., \& Hanson, R. 1994, NASA Ames Research Center, Tech. Rep. FIA-94-12

Dekeyser, F. 2001, Restauration de séquences d'images par des approches spatiotemporelles, Ph.D. Thesis, Université de Rennes I, France

Elad, M., \& Feuer, A. 1997, IEEE Trans. Image Process., 6, 1646

Elad, M., \& Hel-Or, Y. 2001, IEEE Trans. Image Process., 10, 1187

Farsiu, S., Robinson, D., Elad, M., \& Milanfar, P. 2004, Int. J. Imaging Systems and Technology, 14, 47

Irani, M., \& Peleg, S. 1991, Graphical Models and Image Processing (CVGIP), 53, 231

Kjeldsen, H., \& Frandsen, S. 1992, PASP, 104, 413

Lauer, T. R. 1999, PASP, 111, 227

Matthews, J. M., Kuschnig, R., Guenther, D. B., et al. 2004, Nature, 430, 51

Mighell, K. J. 1999, Algorithms for CCD Stellar Photometry, Astronomical Data Analysis Software and Systems VIII, ASP Conf. Ser., 172

Mo, J., \& Hanisch, R. J. 1995, Restoration of HST WFPC2 Images in Gyro-Hold Mode, Astronomical Data Analysis Software and Systems IV, ASP Conf. Ser., 77

Nguyen, N., Milanfar, P., \& Golub, G. H. 2001, IEEE Trans. Image Process., 10, 573

Park, S. C., Park, M., \& Kang, M. 2003, IEEE Signal Processing Mag., 21

Walker, G., Matthews, J., Kuschnig, R., et al. 2003, PASP, 115

Yang, C., Duraiswami, R., \& Davis, L. 2002, IEEE Int. Conf. Image and Graphics

Zomet, A., \& Peleg, S. 2001, Super-Resolution From Multiple Images Having Arbitrary Mutual Motion, ed. S. Chaudhuri, Super-Resolution Imaging (Kluwer Academic Press) 